\title{
Erratum to: Population pharmacokinetic modelling of doxorubicin and doxorubicinol in children with cancer: is there a relationship with cardiac troponin profiles?
}

\author{
Kuhan Kunarajah ${ }^{1}$ - Stefanie Hennig ${ }^{2}$ (1) - Ross L. G. Norris ${ }^{2,3,4,7}$ - Michael Lobb ${ }^{3}$. \\ Bruce G. Charles ${ }^{2} \cdot$ Ross Pinkerton $^{5} \cdot$ Andrew S. Moore ${ }^{1,5,6}$
}

Published online: 8 June 2017

(C) Springer-Verlag Berlin Heidelberg 2017

\section{Erratum to: Cancer Chemother Pharmacol DOI 10.1007/s00280-017-3309-6}

The author would like to correct the errors in the Appendix in the original publication of the article. The correct Appendix is given in the following page. The original article was corrected.

The online version of the original article can be found under doi:10.1007/s00280-017-3309-6.

Andrew S. Moore

andrew.moore@uq.edu.au

1 Translational Research Institute, The University of Queensland Diamantina Institute, Brisbane, Australia

2 School of Pharmacy, Pharmacy Australia Centre of Excellence (PACE), The University of Queensland, Brisbane, Australia

3 Mater Medical Research Institute, The University of Queensland, Brisbane, Australia

4 School of Pharmacy, Griffith University, Gold Coast, Australia

5 Oncology Services Group, Children's Health Queensland Hospital and Health Service, Brisbane, Australia

6 UQ Child Health Research Centre, The University of Queensland, Brisbane, Australia

7 Present Address: Department of Clinical Pharmacology, St. Vincent's Hospital, Sydney, NSW, Australia 


\section{Appendix: NMTRAN control stream for the final model}

\$PROBLEM DOX PK

\$INPUT COM ID DAT1=DROP TIME AMT RATE DV

MDV FLAG CMT BQL OCC WT AGE SEX HT BSA BSTM

PSTM INFRATE PCAMT CPCAMT

SDATA DoxData SH14.csv IGNORE=\#

\$SUBROUTINE ADVAN13 TRANS1 TOL $=3$

\$MODEL NCOMP $=5$

$$
\begin{aligned}
& \text { COMP(CENT) } \\
& \text { COMP(PER1) } \\
& \text { COMP(PER2) } \\
& \text { COMP(MET) } \\
& \text { COMP(TROP) }
\end{aligned}
$$

\$PK

;;------ COVARIATES -

TBASEPCAMT $=$ THETA $(21) *($ PCAMT -90$)$

FBSACL $=(1+($ BSA-1.8)*(THETA $(10)))$

FAGE $=\left(1+(\mathrm{AGE} / 8.4)^{* *}\right.$ THETA $\left.(9)\right)$

TVCL $=$ THETA $(1) *$ FAGE*FBSACL

TVV1 $=$ THETA $(2) *$ FBSACL

TVQ2 $=$ THETA $(5) *$ FBSACL

TVV2 $=$ THETA $(6) *$ FBSACL

TVQ3 $=$ THETA $(7) *$ FBSACL

TVV3 $=$ THETA $(8) *$ FBSACL

TVCLM $=$ THETA $(11) *$ FBSACL

TVV4 $=$ THETA $(12) *$ FBSACL

TVQM $=$ THETA $(13) *$ FBSACL

;;---- Trop model

TVKDEG $=$ THETA $(16)$

TVTBASE $=$ THETA $(17) *(1+$ TBASEPCAMT $)$

TVEMAX $=$ THETA $(19)$

TVEC50 $=$ THETA $(20$

;;-------- Between subject variability --------

$\mathrm{IIVCL}=\mathrm{ETA}(1)$

IIVCLM $=\operatorname{ETA}(2)$

$\mathrm{IIVQM}=\mathrm{ETA}(3)$

IIVQ3 $=\mathrm{ETA}(4)$

IIVV3 $=$ ETA(5)

IIVTBASE $=$ ETA $(6)$

;;------------ parameter estimates ---------

$\mathrm{CL}=\mathrm{TVCL} * \operatorname{EXP}(\mathrm{IIVCL})$

$\mathrm{V} 1=\mathrm{TVV} 1$

$\mathrm{Q} 2=\mathrm{TVQ} 2$

$\mathrm{V} 2=\mathrm{TVV} 2$

$\mathrm{Q} 3=\mathrm{TVQ} 3 * \operatorname{EXP}(\mathrm{IIVQ3})$

$\mathrm{V} 3=$ TVV $3 *$ EXP(IIVV3)

CLM $=$ TVCLM $^{*}$ EXP(IIVCLM)

$\mathrm{V} 4=\mathrm{TVV} 4$

$\mathrm{QM}=\mathrm{TVQM}^{*} \mathrm{EXP}(\mathrm{IIVQM})$

EC50 $=$ TVEC50

EMAX $=$ TVEMAX

KDEG $=$ TVKDEG

TBASE $=$ TVTBASE $*$ EXP(IIVTBASE $)$

KSYN $=$ TBASE* KDEG

K12=Q2/V1

$\mathrm{K} 21=\mathrm{Q} 2 / \mathrm{V} 2$

$\mathrm{K} 13=\mathrm{Q} 3 / \mathrm{V} 1$

$\mathrm{K} 31=\mathrm{Q} 3 / \mathrm{V} 3$

$\mathrm{K} 10=\mathrm{CL} / \mathrm{V} 1$

$\mathrm{K} 14=\mathrm{QM} / \mathrm{V} 1$

$\mathrm{K} 41=0$

$\mathrm{K} 40=\mathrm{CLM} / \mathrm{V} 4$

$\mathrm{S} 1=\mathrm{V} 1$

$\mathrm{S} 4=\mathrm{V} 4$

$\mathrm{S} 5=1$

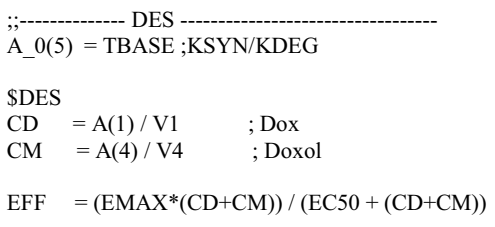

IPRED $=0$

IF (CMT.EQ.1) THEN ;Dox

IPRED $=$ DOX

$\mathrm{W}=\operatorname{SQRT}((\operatorname{THETA}(3) * \operatorname{IPRED}) * * 2+\operatorname{THETA}(4) * * 2)$

ENDIF

IF (CMT.EQ.4)THEN ;Doxol

IPRED $=$ MET

$\mathrm{W}=\mathrm{SQRT}((\operatorname{THETA}(14) * \operatorname{IPRED}) * * 2+\operatorname{THETA}(15) * * 2)$

ENDIF

IF (CMT.EQ.5)THEN ;cTnI

IPRED $=$ TROP

$\mathrm{W}=$ THETA $(18) *$ IPRED

ENDIF

IRES=DV-IPRED

IF(W.EQ.0) $\mathrm{W}=1$

IWRES=IRES/W

$\mathrm{Y}=\operatorname{IPRED}+\mathrm{W}^{*} \mathrm{EPS}(1)$

;;-----INITIALS -

\$THETA

$(0,58.7)$

$(0,32.2)$

$(0,0.203)$

$(0,0.238)$

$(0,35.8)$ FIX

$(0,3810)$ FIX

$(0,65.1)$ FIX

$(0,705)$ FIX

$(0,0.736)$ FIX

$\quad ; 11$ CLM

$\quad ; 12 \mathrm{~V} 4$

$(0,32.1)$ FIX $\quad ; 13 \mathrm{Qm}$

$(0,0.229) \quad ; 14$ Prop.RE (sd) Doxol

$(0,0.954) \quad ; 15$ Add.RE (sd) Doxol

$(0,0.587) \quad ; 16 \mathrm{Kdeg}$ Trop $\mathrm{k}=0.3 \sim \mathrm{t} 1 / 2=2$ hours $\sim$ steady state $\sim 8-10 \mathrm{~h}$

$(0,20.5) \quad ; 17$ TBASE

$(0,0.582) \quad ; 18$ Prop.RE trop (sd) cTnI

$(0,0.154) \quad ; 19$ EMAX

$(0,11.8) \quad ; 20 \mathrm{EC} 50 \mathrm{CD}+\mathrm{CM}$

$(0,0.00308) \quad ; 21$ TBASEPCAMT

SOMEGA BLOCK(2)

$0.0344 \quad ; 1 \mathrm{BSV}$ CL

$0.05230 .116 \quad ; 2 \mathrm{BSV}$ CLM

\$OMEGA $0.11 \quad ; 3$ BSV OM

SOMEGA $0.0497 \quad ; 4$ BSV Q3

SOMEGA $0.0241 ; 5 \mathrm{BSV}$ V3

\$OMEGA 0.0607 ;6 BSV TBASE

\$SIGMA 1 FIX ; Residual error

\$ESTIMATION METHOD=1 INTER MAXEVAL=9999 NOABORT SIG=3 PRINT=1 \$COVARIANCE 